\title{
Projecting genetic diversity and population viability for the fragmented orang-utan population in the Kinabatangan floodplain, Sabah, Malaysia
}

\author{
Michael W. Bruford ${ }^{1, *}$, Marc Ancrenaz ${ }^{2}$, Lounès Chikhi ${ }^{3}$, \\ Isabelle Lackman-Ancrenaz ${ }^{2}$, Mahedi Andau ${ }^{4, \mp}$, Laurentius Ambu ${ }^{4}$, \\ Benoît Goossens ${ }^{1}$ \\ ${ }^{1}$ School of Biosciences, Cardiff University, Cathays Park, Cardiff CF10 3TL, UK \\ ${ }^{2}$ Kinabatangan Orang-utan Conservation Project, Sukau, Sabah, Malaysia \\ ${ }^{3}$ Laboratoire Evolution et Diversité Biologique, UMR CNRS 5174, Université Paul Sabatier, Toulouse, France \\ ${ }^{4}$ Sabah Wildlife Department, Kota Kinabalu, Sabah, Malaysia
}

\begin{abstract}
Genetic management of fragmented populations poses logistical and theoretical challenges to conservation managers. Simulating changes in genetic diversity and differentiation within and among fragmented population units under different management scenarios has until now rarely used molecular marker data collected from present-day populations. Here we examine the genetic implications of management options for the highly fragmented yet globally significant orang-utan population in the Lower Kinabatangan Wildlife Sanctuary, Sabah, Malaysia. We simulated the effects of non-intervention, translocation, corridor establishment and a mixture of the latter 2 approaches on future genetic diversity in this population using the stochastic simulation software VORTEX and a well-described molecular dataset for 200 individuals from within the Sanctuary. We found that nonintervention resulted in high extinction risks for a number of subpopulations over short demographic timescales ( $<5$ generations). Furthermore, the exclusive use of either translocation or corridor establishment as a management tool was insufficient to prevent substantial levels of inbreeding using demographically and logistically feasible translocation rates and was insufficient to prevent inbreeding and extinction in the most isolated subpopulations using conservative corridor establishment rates. Instead, a combination of modest translocation rates (1 ind. every 20 yr) and corridor establishment enabled even the most isolated subpopulations to retain demographic stability and constrain localised inbreeding to levels below a threshold of 0.1. Our simulations suggest that this mixed management approach is both a pragmatic and potentially successful course of action and that this combination may be useful in other species and fragmented populations in the future. The use of presentday molecular data in stochastic simulations requires further development, but here we show that it can aid predictive modelling.
\end{abstract}

KEY WORDS: Population modeling - VORTEX - Orang-utan - Kinabatangan - Fragmentation • Genetic diversity

Resale or republication not permitted without written consent of the publisher

\section{INTRODUCTION}

The relative importance of genetic diversity in population viability and management has been debated over the last few decades (e.g. Asquith 2001). While it is now widely accepted that demographic isolation and subsequent inbreeding can potentially be implicated in population extinction (e.g. Saccheri et al. 1998) and that the introduction of unrelated genomes into inbred populations can alleviate such problems (Madsen et al. 1999, 2004, Tallmon et al. 2004, Schwartz \& Mills 2005), the role of genetics in population viability analysis remains limited, and the integration of molecular data is still at an early stage (Reed et al. 2002).

${ }^{\ddagger}$ This paper is dedicated to the memory of Mahedi Andau 
Ongoing population fragmentation is a major issue for many threatened species, and integrated management of subpopulations is expected to play an increasing role in conservation measures. The dynamics of genetic change in fragmented populations is therefore a major focus of attention (e.g. Keyghobadi et al. 2005). However, the consequences of, and optimal strategies for genetic management of metapopulations remain understudied. The relationship between levels of inbreeding and inbreeding depression itself are still debated (Keller \& Waller 2002) and are likely to be case specific. Issues such as the frequency and symmetry of genetic exchange and its relationship to the number of subpopulations managed (e.g. Bouchy et al. 2005), the dynamics of incorporation of immigrant genes into recipient subpopulations (Keller et al. 2001, Saccheri \& Brakefield 2002) plus the potential negative consequences of immigration and the role of population size on genetic load (Robert et al. 2003, Tallmon et al. 2004) are areas in which more research is required if genetic management is to be improved. One area largely unexplored in this context is the integration of molecular population genetics into population viability models and the way in which genetic diversity is predicted to evolve, using marker data as a starting point, under different management strategies.

The increasing use of molecular data to measure genetic variation in threatened populations potentially facilitates key management decisions (e.g. Frankham 2003, Talbot et al. 2003, Tallmon et al. 2004). However, using molecular data directly in predictive population viability analysis remains limited. The need to integrate analysis of past, current and future parameter trajectories in conservation is acknowledged (e.g. Bowen \& Roman 2005). When coupled with the observation that rapid evolution in small populations can impact endangered species (Stockwell et al. 2003), realistic predictive simulations of future genetic diversity becomes a potentially important tool in population management.

Using coalescent-based genealogical simulations and contemporary molecular data, we previously reported evidence for a recent demographic collapse and the resultant contemporary genetic structure of the now highly fragmented orang-utan population in the Lower Kinabatangan Wildlife Sanctuary in Sabah, Malaysia (Goossens et al. 2005, 2006a,b, Jalil et al. 2008). Here, we used these data to simulate future trends in genetic diversity and demographic viability by stochastic population modelling of alternative management scenarios for this metapopulation. We tested the effects of managed translocation and the establishment of habitat corridors and their consequences on genetic diversity and demographic viability within and in this series of well-characterised, fragmented sub- populations (Ancrenaz et al. 2004). We address the predicted effects on genetic diversity of translocations, the establishment of forest fragment corridors and a mixed approach combining the two. We compare these results to no intervention, assessing the influence of the above factors on the trajectory of genetic diversity in the subpopulations concerned.

\section{MATERIALS AND METHODS}

Study site, population status and data. The orangutan population of the Lower Kinabatangan Wildlife Sanctuary (LKWS) is one of the most well-studied on the island of Borneo (e.g. Lackman-Ancrenaz et al. 2001, Ancrenaz 2003, 2004). The sanctuary lies in the floodplain of the Kinabatangan River, one of the most important wetlands in Malaysia for biodiversity despite high levels of habitat degradation and fragmentation. This degradation is manifest in different sized areas of partially degraded and fragmented forest along both banks of the river (Lackman-Ancrenaz et al. 2001, Ancrenaz 2003) largely surrounded by oil palm plantations. Despite this, LKWS supports a very high density of primates. The Sabah State Government officially gazetted 10 forest lots totalling about 26000 ha on 11 August 2005, aiming to create a forest corridor along the river linking 7 Virgin Jungle Forest Reserves (VJFRs) totalling approximately 15000 ha, and state and private lands (about 10000 ha) currently under forest cover (Fig. 1; Ancrenaz et al. 2004). Ancrenaz et al. (2004) estimated that the forest in the Lower Kinabatangan floodplain comprised approximately 52000 ha in 2001, of which 41000 ha were judged potentially suitable for orang-utans by a subsequent aerial survey. This study, using both ground and helicopter-based surveys, estimated 1125 (range: 686-1883) orang-utans in the area, comprising 670 (394-1158) in the LKWS, 228 (153-351) in VJFRs and 227 (139-374) in non-protected forest connected either to the LKWS or to the forest reserves.

The Kinabatangan population can no longer be considered a single unit in its current form (Lackman-Ancrenaz et al. 2001). To estimate population sizes, Ancrenaz et al. (2004) designated 11 'primary sampling units' (PSUs), where each PSU comprised one lot of the sanctuary and connected forests, irrespective of their administrative status (VJFR, private or state land, Fig. 1). Two PSUs were designated for Lot 10, since it is divided into 2 separate forest patches. The authors then carried out line transect nest surveys (4 to $28 \mathrm{PSU}^{-1}$; Ancrenaz et al. 2004). Forest type within each PSU was classified according to the level of disturbance: heavily disturbed, slightly disturbed and undisturbed. Orang-utan densities were then estimated from nest densities (Ancrenaz 


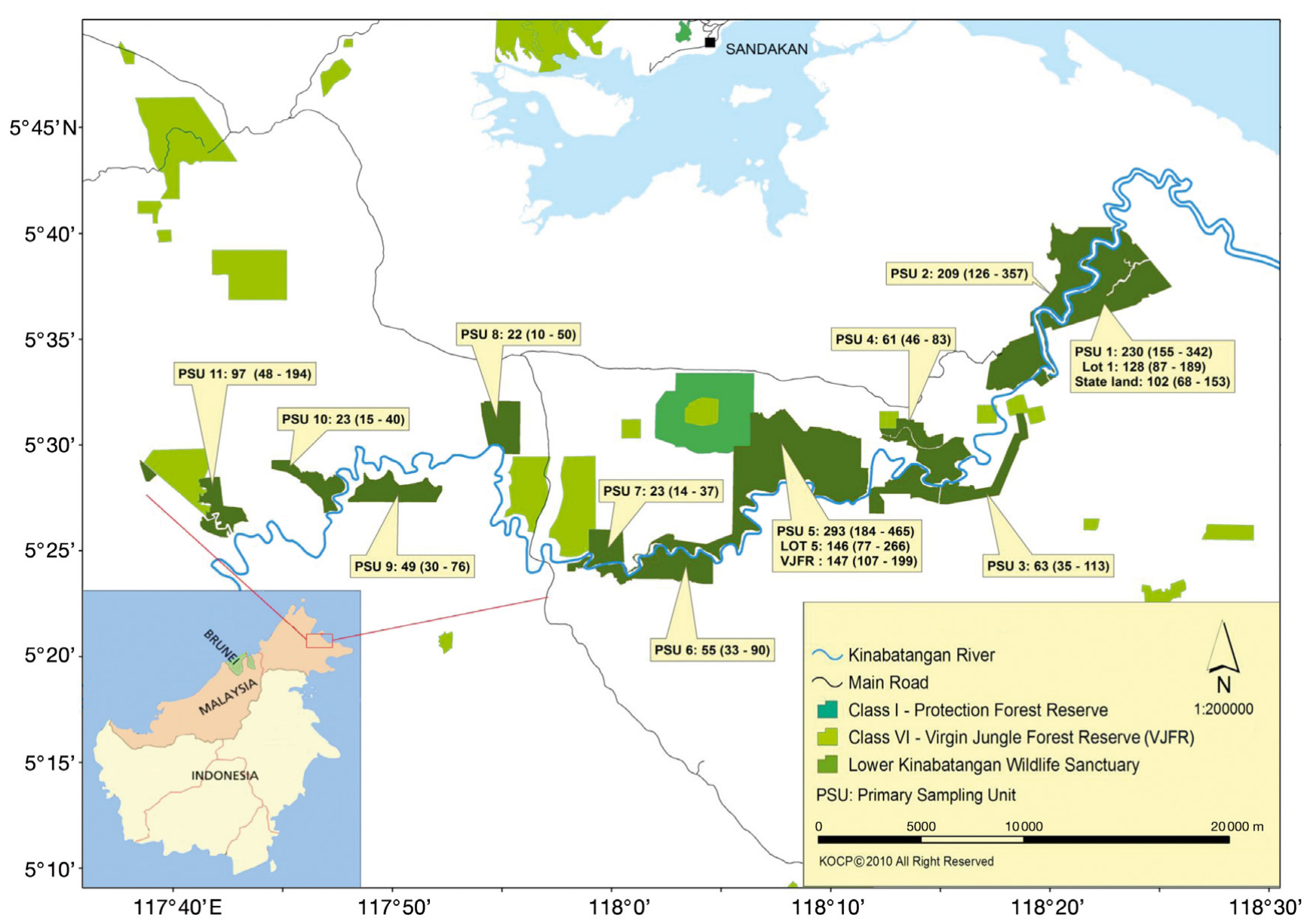

Fig. 1. Kinabatangan region and the 11 primary sampling units (PSUs). Values indicate mean no. of orang-utans estimated to occur in the respective PSUs in 2004 (range in parantheses). Reprinted from Ancrenaz et al. (2004a), with permission from Cambridge University Press

et al. 2004, their Table 2) using the software Distance v3.5. The 11 PSUs were grouped into 9 sampled populations by Goossens et al. $(2005,2006 a, b)$ corresponding to sampling units (S1 to S9).

Molecular analysis using microsatellite genotypes from faecal DNA of 200 individuals sampled from all regions of the LKWS (Goossens et al. 2000) confirmed the signature of a demographic decline of 2 orders of magnitude over the last 200 yr (Goossens et al. 2006a). Although estimates of genetic diversity were superficially high (e.g. an expected heterozygosity of 0.74 ; Goossens et al. 2005), evidence was found for significant genetic differentiation between most PSUs (average $F_{\mathrm{ST}} 0.04, \mathrm{p}<0.001$ ) and was significantly higher across the Kinabatangan River than on the same river side (Goossens et al. 2005). Bayesian migration estimates and assignment tests indicated that gene flow was highly unlikely to occur across the river. In contrast, migration between lots on the same side of the river had a high probability, indicating that orangutans recently moved relatively freely between neigh- bouring areas, consistent with the recent history of logging and subsequent oil palm agriculture since the 1970s and 1980s.

We analysed molecular and demographic data using the stochastic population modelling program VORTEX (v 9.61, Miller \& Lacy 2005, Lacy et al. 2005) to model the Lower Kinabatangan population using parameter values derived from observation and research in LKWS (e.g. Ancrenaz et al. 2004), from a previous orang-utan workshop carried out at in Kota Kinabalu, Malaysia, in August 2003 (Ancrenaz 2003) and an orang-utan population and habitat viability assessment (PHVA) carried out in Jakarta, Indonesia, in January 2004 (Singleton et al. 2004; Appendix 1). During the latter workshop, 1 of the 2 modelling working groups focused on the Bornean orang-utan, which has markedly distinct life history parameters from the Sumatran species. Much of our data are collated from this exercise. The approach was to derive a baseline model for a 'typical' Bornean orang-utan population based on the consensus data for the 2004 meeting (Singleton et al. 2004). 
General parameters of the model. One thousand simulations were carried out for each model, and results were compared for the following time periods: (1) $25 \mathrm{yr}$, which corresponds to between 1 and 3 orangutan generation times (depending on the estimate used) and a potential timescale for a short-term management plan; (2) $100 \mathrm{yr}$, equivalent to 4 to 12 generations; and (3) a long-term period of $250 \mathrm{yr}$, equivalent to 10 to 30 orang-utan generations. General parameters used, are not detailed here, can be found in Appendix 1.

Inbreeding depression was incorporated into some models using a lethal equivalents estimate of 4.06 genome $^{-1}$ derived from captive orang-utan data (Singleton et al. 2004), and environmental variance in reproduction was not correlated to that of survival. Two types of catastrophe were modelled (Appendix 1) to reflect El Niño-induced severe drought events and occasional human-orang-utan conflict due to crop raiding. Hunting and further habitat loss due to human encroachment were not modelled, although smallscale illegal logging and land conversion on private plots remain periodic issues in the Kinabatangan.

Since the orang-utan population in LKWS is at extremely high density in some PSUs (Ancrenaz et al. 2004), we modelled density-dependent reproduction. The equation that VORTEX uses to model density dependence is (after Lacy et al. 2005):

$$
P(N)=\left(P(0)-\left[(P(0)-P(K))\left(\frac{N}{K}\right)^{B}\right]\right) \frac{N}{N+A}
$$

where $P(N)$ is the percent of females that breed when the population size is $N, P(K)$ is the percent that breed when the population is at carrying capacity $K$, and $P(0)$ is the percent of females breeding when the population is close to 0 in the absence of any Allee effect. $A$ is the magnitude of the Allee effect, and is equivalent to the population size where the percentage of females breeding is $50 \%$ of what it would be in the absence of such an effect (Lacy et al. 2005). The exponent $B$ determines the shape of the curve relating the percent breeding to population size. If $B=1$, the percent breeding changes linearly with population size. If $B=2, P(N)$ is a quadratic function of $N$. In the absence of detailed observational data, we used the latter exponent $(B=2)$ for all simulations. The Allee parameter was not used, since the PSUs are relatively small in size, and orangutans can locate potential mates even at low densities. We modelled the proportion of females breeding at low density as $18 \%$ (equivalent to an inter-birth interval of 5 to $6 \mathrm{yr}$, as observed in the LKWS) and at high density as $12.5 \%$ (equivalent to an inter-birth interval of $8 \mathrm{yr}$, an extreme value for the Kinabatangan).

The population age distribution was estimated according to the results of 71 river sightings where broad age/sex designations were possible, made between 1999 and 2001 (Appendix 1) adjusted for the relative lack of visibility of juveniles. The proportion of adult males:females at 2.42:1 is highly unusual for a primate and has not been reported previously for orang-utans, but here was observed both using river sighting data and a detailed demographic census of a study site within Lot 2 of the LKWS (Ancrenaz et al. 2004). It is possible that this adult sex ratio imbalance reflects sex-biased dispersal rates into the lots from suboptimal habitat in the recent past or when the population first became fragmented. We modelled $100 \%$ of adult males as potential breeders, since Goossens et al. (2006b) found that both large males possessing secondary sexual characters and small males lacking such characters were successful breeders in the Kinabatangan, confirming the results of Utami et al. (2002) on Sumatran orang-utans.

Population-specific parameters. Mortality and reproduction parameters were derived from the data of Singleton et al. (2004) and in the absence of PSUspecific data were applied equally to all PSUs. The only population-specific parameters specified in the model were population size (mean estimates from Ancrenaz et al. 2004) and initial carrying capacity, adjusted from Ancrenaz (2003), who used maximum observed density multipliers for well-defined habitat types where the area of each was characterised for each PSU. Some parameters, such as age-specific mortality and its environmental variation, are unlikely to be accurate for all PSUs because field researchers in Borneo have recorded very few deaths. The 3 mortality schedules that were tested by Singleton et al. (2004) did not differentiate mortality rates between different juvenile and subadult age classes or between sexes. We refined these estimates based on observations over a 10 yr period in PSU 2 (M. Ancrenaz et al. unpubl. data), specifically focusing on refining the estimates for juveniles and dispersing-age subadults. Our mortality estimates are more conservative than previous models. Adjustments were made primarily to reflect relative degradation of the same habitat in each PSU (Ancrenaz et al. 2004). Prior to adjustment, for all LKWS lots, state and private land in PSUs 1 and 2, which are large and equivalent in quality and can support very high densities of orang-utans (up to $6 \mathrm{~km}^{-2}$, the highest ever recorded; Ancrenaz et al. 2004), we used a carrying capacity of $6.5 \mathrm{~km}^{-2}$. For all VJFRs, which comprise mostly limestone primary forest, which supports a lower density of orang-utans (maximum recorded mean $3.8 \mathrm{~km}^{-2}$ for Gomatong VJFR; Ancrenaz et al. 2004), we used a $K$ value of $4.5 \mathrm{~km}^{-2}$.

Model design. Models were designed to test the genetic and demographic consequences of the following management approaches. 
(1) No intervention: PSUs were modelled as separate demographic units for the duration of the simulations. No translocations were modelled and the carrying capacity of the PSUs was kept constant.

(2) Managed translocations - frequency: a single adult female was modelled as being non-reciprocally translocated once every 10, 20 or 50 yr from PSU 2 to both PSUs 4 and 7 and from PSU 5 to PSUs 8, 10 and 11 (north of the river) and for the south side of the river, from PSU 1 to PSUs 3, 6 and 9. The choice of the source populations was made on the basis of their current size and location.

(3) Corridors: here we simulated the effects of no translocation but a gradual increase in carrying capacity $(0.1$ to $2 \%$ per annum, depending on the area of the PSU, its distance from its nearest neighbour and constraints such as rivers and roads, see Appendix 1) to simulate the effects of forest corridors. We conservatively modelled these to establish over $100 \mathrm{yr}$ (PSUs 4 to 5 and 5 to 7 and PSUs 1 to 3 ) or 250 yr (the remainder). Once connected, a conservative value for interPSU dispersal of $5 \%$ of adults per annum was assumed, due to the philopatric nature of both males and females in this population (Goossens et al. 2006b).

(4) Mixed approach: To assess synergistic effects between approaches 2 and 3, we simulated a mixed strategy, where we modelled corridor development as in 3, but included translocation of 1 adult female every 20 yr described in 2.

All models were simulated both with and without inbreeding depression to assess the effects of genetic load on population viability. We report results for the following parameters: mean probability of extinction (proportion of simulations that reached a population size of 0 ), mean final population size, mean gene diversity (heterozygosity expected under Hardy-Weinberg equilibrium), mean inbreeding coefficient and the mean level of genetic differentiation between PSUs (estimated as Wright's $F_{\mathrm{ST}}$ ). The last 3 values were calculated using the mean of 20 randomly chosen output files generated by each simulation (using the genetic analysis subroutine) and subsequently analysed using Genepop v3.4 (Raymond \& Rousset 1995).

\section{RESULTS}

\section{No intervention}

When taking into account the possibility of inbreeding depression, the 'no intervention' models predicted negative growth rates in almost all models and genetic stability (maintenance of $\geq 95 \%$ of extant gene diversity) over $250 \mathrm{yr}$ for only 3 of the 11 PSUs (1, 2 and 5 with $0.954,0.967$ and 0.966 respectively, Table 1).
Models incorporating inbreeding depression are likely to be the more realistic given the now low likelihood of dispersal among PSUs in the absence of managed translocation or habitat corridors linking them. Extinction probabilities after $250 \mathrm{yr}$ of $\geq 5 \%$ were predicted for 4 PSUs $(7,8,9,10$ with 0.122, 0.076, 0.079, 0.349, respectively) under models excluding inbreeding depression and for 6 PSUs $(4,6,7,8,9,10$ with 0.054, $0.123,0.388,0.275,0.276,0.706$, respectively) for models including inbreeding depression. PSU 10 also had a $\geq 5 \%$ extinction probability after $100 \mathrm{yr}$ with and without inbreeding depression (0.069, 0.091, respectively). Inbreeding coefficients after $250 \mathrm{yr}$ ranged from 0.026 (PSU 5, incorporating inbreeding depression) to 0.299 (PSU 10, excluding inbreeding depression; Table 1). Furthermore, mean final population size decreased in 7 of 11 PSUs when inbreeding depression was not included and in 9 of 11 PSUs when inbreeding was included (Table 1). Genetic differentiation among PSUs after $250 \mathrm{yr}$ (Table 2), as estimated by Wright's $F_{\mathrm{ST}}$, increased over present-day values and was significant in all 55 pairwise comparisons, ranging from 0.044 (PSU 5 versus PSU 11) to 0.455 (PSU 6 versus PSU 10; see Table S1a in the supplement for equivalent values at 25 and $100 \mathrm{yr}$; available at www.intres.com/articles/suppl/n012p249_supp_pdf). Significant differentiation among PSUs is also a feature of the present-day microsatellite marker dataset (see Goossens et al. 2005); however, the highest observed pairwise value was 0.12 , with the average being 0.04 over all comparisons and 0.02 among populations from the same side of the river. These simulations therefore predict a major increase in genetic differentiation.

\section{Managed translocations}

We analysed the effects of translocation frequency on genetic diversity and demographic stability choosing females as the translocated sex, since, as all adult males are potential breeders (Goossens et al. 2006b), the sex of translocated individuals made minimal differences to model outcomes (data not shown). As expected for regularly augmented populations, expected gene diversity values were generally higher than for the 'no intervention' model, and no pairwise comparison showed a higher predicted gene diversity for the 'no intervention' model after 250 yr (Table 1, Table S2 available at www.int-res.com/articles/suppl/ n012p249_supp_pdf).

The predicted probability of extinction (P $[E])$ after 250 yr was $<0.01$ for all scenarios except for the PSU 10 for the 1 translocation every $50 \mathrm{yr}$ scenario $(\mathrm{P}[E]=$ 0.12), for PSU 9 for the same translocation regime $(\mathrm{P}[E]=0.03)$ and for PSU 1 (donor population) in the 
Table 1. Basic demographic and genetic parameters for all primary sampling units (PSUs) of the Lower Kinabatangan Wildlife Sanctuary after 25, 100 and 250 yr under a non-intervention management scenario. Means are of 1000 simulations.

$\mathrm{P}(E)$ : probability of extinction; pop: population; GD: genetic diversity; TE: time to extinction

\begin{tabular}{|c|c|c|c|c|c|c|c|c|c|}
\hline PSU & $\begin{array}{l}\text { Carrying } \\
\text { capacity }\end{array}$ & $\begin{array}{l}\text { Mean } \\
(\mathrm{r})\end{array}$ & $\mathrm{SD}$ & $\begin{array}{c}\mathrm{P}(E) \text { after } \\
25 / 100 / 250 \mathrm{yr}\end{array}$ & $\begin{array}{l}\text { Initial/mean } \\
\text { final pop size }\end{array}$ & $\mathrm{SD}$ & $\begin{array}{c}\text { Retained GD after } \\
25 / 100 / 250 \mathrm{yr}\end{array}$ & $\begin{array}{l}\text { Mean } F \text { after } \\
25 / 100 / 250 \mathrm{yr}\end{array}$ & $\begin{array}{l}\text { Mean } \\
\text { TE }\end{array}$ \\
\hline \multicolumn{10}{|c|}{ Without inbreeding depression } \\
\hline PSU 1 & 230 & -0.001 & 0.037 & $0 / 0 / 0$ & $230 / 157.7$ & 27.33 & $0.994 / 0.981 / 0.954$ & 0/0.010/0.036 & 0 \\
\hline PSU 2 & 351 & 0.001 & 0.035 & $0 / 0 / 0$ & $209 / 245.02$ & 39.15 & 0.994/0.985/0.967 & $0 / 0.009 / 0.023$ & 0 \\
\hline PSU 3 & 108 & 0 & 0.045 & 0/0/0.001 & $63 / 71.48$ & 17.24 & 0.980/0.949/0.889 & $0.001 / 0.030 / 0.098$ & 0 \\
\hline PSU 4 & 72 & -0.001 & 0.051 & $0 / 0 / 0.017$ & $61 / 44.36$ & 14.1 & $0.977 / 0.933 / 0.837$ & $0.001 / 0.037 / 0.131$ & 192.2 \\
\hline PSU 5 & 313 & -0.001 & 0.036 & $0 / 0 / 0$ & $293 / 218.63$ & 36.51 & 0.995/0.986/0.966 & 0/0.008/0.027 & 0 \\
\hline PSU 6 & 62 & -0.002 & 0.054 & 0/0.029/0.036 & $55 / 37.72$ & 12.52 & 0.976/0.923/0.808 & $0.001 / 0.041 / 0.150$ & 187.8 \\
\hline PSU 7 & 54 & 0 & 0.063 & $0 / 0.019 / 0.122$ & $23 / 30.93$ & 11.55 & $0.947 / 0.964 / 0.732$ & $0.003 / 0.080 / 0.216$ & 149.5 \\
\hline PSU 8 & 97 & 0.003 & 0.056 & $0 / 0.022 / 0.076$ & $22 / 58.41$ & 19.12 & $0.946 / 0.878 / 0.796$ & $0.003 / 0.079 / 0.174$ & 137.5 \\
\hline PSU 9 & 49 & -0.003 & 0.06 & $0 / 0.006 / 0.079$ & $49 / 28.45$ & 10.91 & $0.971 / 0.900 / 0.760$ & $0.001 / 0.052 / 0.204$ & 185.2 \\
\hline PSU 10 & 31 & -0.003 & 0.075 & $0 / 0.069 / 0.349$ & $23 / 17.13$ & 7.48 & $0.944 / 0.827 / 0.628$ & $0.002 / 0.089 / 0.299$ & 152.8 \\
\hline PSU 11 & 120 & -0.001 & 0.043 & $0 / 0 / 0$ & $97 / 79.02$ & 19.33 & $0.987 / 0.966 / 0.906$ & $0.001 / 0.021 / 0.076$ & 0 \\
\hline \multicolumn{10}{|c|}{ Including inbreeding depression } \\
\hline PSU 1 & 230 & -0.002 & 0.037 & $0 / 0 / 0$ & 230/144.39 & 30.32 & 0.994/0.981/0.952 & 0/0.009/0.036 & 0 \\
\hline PSU 2 & 351 & 0 & 0.035 & $0 / 0 / 0$ & $209 / 227.16$ & 39.45 & 0.994/0.985/0.966 & 0/0.008/0.026 & 0 \\
\hline PSU 3 & 108 & -0.001 & 0.046 & 0/0/0.016 & $63 / 52.84$ & 19.55 & $0.980 / 0.949 / 0.874$ & $0.001 / 0.025 / 0.097$ & 216 \\
\hline PSU 4 & 72 & -0.004 & 0.054 & 0/0.001/0.068 & $61 / 30.43$ & 14.61 & 0.979/0.933/0.808 & $0.001 / 0.030 / 0.133$ & 198.1 \\
\hline PSU 5 & 313 & -0.001 & 0.036 & $0 / 0 / 0$ & $293 / 203.83$ & 38.07 & $1.000 / 0.986 / 0.965$ & 0/0.006/0.026 & 0 \\
\hline PSU 6 & 62 & -0.005 & 0.058 & $0 / 0.005 / 0.123$ & $55 / 24.37$ & 12.16 & $0.976 / 0.923 / 0.780$ & $0.001 / 0.039 / 0.155$ & 197.4 \\
\hline PSU 7 & 54 & -0.004 & 0.069 & 0/0.032/0.388 & 23/18.97 & 10.53 & $0.947 / 0.963 / 0.702$ & $0.002 / 0.069 / 0.213$ & 173.8 \\
\hline PSU 8 & 97 & -0.002 & 0.063 & $0 / 0.038 / 0.275$ & $22 / 33.37$ & 19.31 & $0.946 / 0.872 / 0.762$ & $0.002 / 0.065 / 0.161$ & 167.4 \\
\hline PSU 9 & 49 & -0.006 & 0.065 & $0 / 0.008 / 0.274$ & 49/18.1 & 10.17 & $0.971 / 0.900 / 0.726$ & $0.001 / 0.050 / 0.189$ & 194.1 \\
\hline PSU 10 & 31 & -0.008 & 0.08 & 0/0.091/0.706 & 23/10.16 & 6.37 & 0.944/0.874/0.611 & $0.002 / 0.080 / 0.288$ & 164.2 \\
\hline PSU 11 & 120 & -0.002 & 0.043 & 0/0/0.004 & $97 / 64.03$ & 20.89 & 0.987/0.966/0.897 & $0.002 / 0.020 / 0.076$ & 203.8 \\
\hline
\end{tabular}

1 translocation every 10 yr scenario $(\mathrm{P}[E]=0.02)$. When comparing the predicted mean final population size for models incorporating inbreeding depression (Tables 1 \& 3) an increase for recipient populations of $82.21 \pm$ $34.53 \%$ (SD) for 1 female every $10 \mathrm{yr}, 53 \pm 21.63 \%$ for 1 female every $20 \mathrm{yr}$ and $11.87 \pm 5.8 \%$ for 1 female every 50 yr was recorded. However, this was accompanied by a concomitant donor population decline of $27.71 \pm 17.68 \%, 10.92 \pm 6.2 \%$ and $1.88 \pm 1.75 \%$, respectively. Within-population inbreeding coefficients after $250 \mathrm{yr}$ simulations were reduced in all recipient populations when compared to the no-inter-

Table 2. Wright's $F_{\mathrm{ST}}$ values in 2001 (above diagonal) and values predicted after $250 \mathrm{yr}$ (below diagonal) for the non-intervention model. Simulation results, estimated using Genepop v3.4, are the means of 20 randomly chosen surviving simulations. Predicted values at Years 25 and 100 in the model are displayed in Table S1a in the supplement available at www.int-res.com/articles/ suppl/n012p249_supp.pdf. The 2001 data (taken from Goossens et al. 2005) combine primary sampling units (PSUs) 5 and 7 and PSUs 10 and 11: identical allele frequencies were therefore allocated to combined PSUs for simulation purposes. All comparisons in the present-day data set were significant at the $5 \%$ level or less except PSU 2 versus 8 ; PSUs 5 and 7 versus 8 ; and PSUs 10 and 11 versus 8 . All values for the simulated models were significant at the $5 \%$ level or less

\begin{tabular}{|c|c|c|c|c|c|c|c|c|c|c|c|}
\hline & PSU 1 & PSU 2 & PSU 3 & PSU 4 & PSU 5 & PSU 6 & PSU 7 & PSU 8 & PSU 9 & PSU 10 & PSU 11 \\
\hline PSU 1 & & 0.051 & 0.029 & 0.056 & 0.040 & 0.015 & 0.040 & 0.075 & 0.022 & 0.045 & 0.045 \\
\hline PSU 2 & 0.087 & & 0.074 & 0.038 & 0.014 & 0.037 & 0.014 & 0.022 & 0.061 & 0.042 & 0.042 \\
\hline PSU 3 & 0.124 & 0.124 & & 0.092 & 0.067 & 0.028 & 0.067 & 0.120 & 0.027 & 0.079 & 0.079 \\
\hline PSU 4 & 0.194 & 0.128 & 0.222 & & 0.013 & 0.046 & 0.013 & 0.029 & 0.065 & 0.019 & 0.019 \\
\hline PSU 5 & 0.072 & 0.041 & 0.102 & 0.114 & & 0.028 & - & 0.014 & 0.054 & 0.015 & 0.015 \\
\hline PSU 6 & 0.133 & 0.130 & 0.095 & 0.208 & 0.121 & & 0.028 & 0.049 & 0.018 & 0.033 & 0.033 \\
\hline PSU 7 & 0.180 & 0.212 & 0.258 & 0.241 & 0.159 & 0.236 & & 0.029 & 0.065 & 0.019 & 0.019 \\
\hline PSU 8 & 0.196 & 0.158 & 0.324 & 0.252 & 0.133 & 0.229 & 0.266 & & 0.079 & 0.020 & 0.020 \\
\hline PSU 9 & 0.188 & 0.202 & 0.199 & 0.287 & 0.221 & 0.247 & 0.331 & 0.301 & & 0.038 & 0.038 \\
\hline PSU 10 & 0.235 & 0.227 & 0.302 & 0.313 & 0.207 & 0.455 & 0.308 & 0.363 & 0.411 & & - \\
\hline PSU 11 & 0.069 & 0.074 & 0.122 & 0.142 & 0.044 & 0.188 & 0.154 & 0.209 & 0.314 & 0.270 & \\
\hline
\end{tabular}


Table 3. Separate effects of managed translocation and corridor establishment on inbreeding and population size in models incorporating inbreeding depression at Years 25, 100 and 250. Note that primary sampling units (PSUs) 1, 2 and 5 are donor populations for translocation purposes (italics)

\begin{tabular}{|c|c|c|c|c|c|c|c|}
\hline \multirow[t]{2}{*}{ Translocation rate } & \multirow[t]{2}{*}{ PSU } & \multicolumn{3}{|c|}{ Inbreeding coefficient -} & \multicolumn{3}{|c|}{$\longrightarrow$ Mean population size } \\
\hline & & Year 25 & Year 100 & Year 250 & Year 25 & Year 100 & Year 250 \\
\hline \multirow[t]{11}{*}{1 adult female every $10 \mathrm{yr}$} & PSU 1 & 0 & 0.010 & 0.046 & 173.47 & 136.96 & 98.35 \\
\hline & $P S U 2$ & 0 & 0.008 & 0.028 & 206.95 & 219.31 & 202.96 \\
\hline & PSU 3 & 0 & 0.020 & 0.046 & 69.52 & 78.63 & 75.7 \\
\hline & PSU 4 & 0 & 0.020 & 0.050 & 57.23 & 54.07 & 50.72 \\
\hline & PSU 5 & 0 & 0.007 & 0.029 & 236.4 & 198.3 & 163.85 \\
\hline & PSU 6 & 0 & 0.024 & 0.053 & 49.71 & 46.87 & 44.53 \\
\hline & PSU 7 & 0.001 & 0.036 & 0.058 & 30.58 & 39.86 & 39.47 \\
\hline & PSU 8 & 0.001 & 0.038 & 0.059 & 32.5 & 60.88 & 66.85 \\
\hline & PSU 9 & 0 & 0.027 & 0.054 & 40.27 & 37.48 & 36.23 \\
\hline & PSU 10 & 0.001 & 0.035 & 0.061 & 24.73 & 24.09 & 23.79 \\
\hline & PSU 11 & 0 & 0.014 & 0.040 & 91.63 & 89.71 & 83.96 \\
\hline \multirow[t]{11}{*}{1 adult female every $20 \mathrm{yr}$} & $P S U 1$ & 0 & 0.009 & 0.040 & 177.54 & 151.41 & 122.73 \\
\hline & PSU 2 & 0 & 0.008 & 0.027 & 208.81 & 231.39 & 215.45 \\
\hline & PSU 3 & 0 & 0.022 & 0.060 & 67.45 & 72.55 & 67.07 \\
\hline & PSU 4 & 0.001 & 0.024 & 0.073 & 56.21 & 49.48 & 44.13 \\
\hline & PSU 5 & 0 & 0.001 & 0.028 & 239.74 & 211.09 & 185.86 \\
\hline & PSU 6 & 0 & 0.028 & 0.076 & 48.81 & 42.49 & 37.59 \\
\hline & PSU 7 & 0.002 & 0.044 & 0.091 & 28.69 & 33.84 & 31.58 \\
\hline & PSU 8 & 0.002 & 0.046 & 0.087 & 30.29 & 49.35 & 54.9 \\
\hline & PSU 9 & 0.001 & 0.0323 & 0.087 & 39.4 & 32.79 & 29.79 \\
\hline & PSU 10 & 0.002 & 0.048 & 0.088 & 23.58 & 20.17 & 18.62 \\
\hline & PSU 11 & 0 & 0.016 & 0.052 & 90.73 & 83.29 & 76.12 \\
\hline \multirow[t]{11}{*}{1 adult female every $50 \mathrm{yr}$} & PSU 1 & 0 & 0.009 & 0.039 & 181.01 & 157.92 & 139.42 \\
\hline & PSU 2 & 0 & 0.008 & 0.027 & 213.24 & 235.48 & 225.41 \\
\hline & PSU 3 & 0. & 0.025 & 0.077 & 66.48 & 69.24 & 59.05 \\
\hline & PSU 4 & 0 & 0.029 & 0.097 & 54.75 & 45.98 & 35.15 \\
\hline & $P S U 5$ & 0 & 0 & 0.027 & 244.02 & 216.79 & 199.84 \\
\hline & PSU 6 & 0.001 & 0.032 & 0.111 & 47.57 & 38.77 & 29.45 \\
\hline & PSU 7 & 0.002 & 0.058 & 0.147 & 27.46 & 28.4 & 22.92 \\
\hline & PSU 8 & 0.002 & 0.058 & 0.129 & 28.47 & 41.29 & 41.8 \\
\hline & PSU 9 & 0 & 0.039 & 0.137 & 38.69 & 29.97 & 21.54 \\
\hline & PSU 10 & 0.001 & 0.063 & 0.170 & 22.32 & 16.95 & 12.65 \\
\hline & PSU 11 & 0 & 0.017 & 0.068 & 89.49 & 79.32 & 68.51 \\
\hline \multirow[t]{11}{*}{ Corridor establishment } & $P S U 1$ & 0 & 0.010 & 0.024 & 183.5 & 171.83 & 183.15 \\
\hline & PSU 2 & 0 & 0.009 & 0.024 & 217.88 & 270.35 & 295.32 \\
\hline & PSU 3 & 0.001 & 0.030 & 0.042 & 65.88 & 95.21 & 198.87 \\
\hline & PSU 4 & 0.001 & 0.033 & 0.042 & 55.27 & 61.84 & 79.03 \\
\hline & PSU 5 & 0 & 0.007 & 0.016 & 247.7 & 235.98 & 258.05 \\
\hline & PSU 6 & 0.001 & 0.038 & 0.109 & 48.93 & 58.77 & 101.02 \\
\hline & PSU 7 & 0.002 & 0.077 & 0.051 & 24.57 & 30.86 & 43.18 \\
\hline & PSU 8 & 0.003 & 0.080 & 0.168 & 25.55 & 51.61 & 109.55 \\
\hline & PSU 9 & 0.001 & 0.046 & 0.117 & 42.26 & 57.5 & 121.91 \\
\hline & PSU 10 & 0.002 & 0.087 & 0.199 & 22.61 & 34.71 & 53.55 \\
\hline & PSU 11 & 0 & 0.020 & 0.046 & 96.42 & 154.38 & 242.19 \\
\hline \multirow[t]{11}{*}{ Mixed approach } & PSU 1 & 0 & 0.011 & 0.038 & 178.87 & 164.19 & 157.22 \\
\hline & PSU 2 & 0 & 0.010 & 0.026 & 209.63 & 257.36 & 284.36 \\
\hline & PSU 3 & 0.001 & 0.025 & 0.053 & 69.94 & 108.83 & 198.7 \\
\hline & PSU 4 & 0.001 & 0.029 & 0.070 & 57.68 & 67.76 & 76.44 \\
\hline & PSU 5 & 0 & 0.008 & 0.027 & 239.78 & 226.08 & 224.99 \\
\hline & PSU 6 & 0.001 & 0.033 & 0.072 & 51.42 & 66.92 & 115.24 \\
\hline & PSU 7 & 0.003 & 0.057 & 0.104 & 28.6 & 39.24 & 42.8 \\
\hline & PSU 8 & 0.002 & 0.056 & 0.091 & 30.06 & 70.36 & 133.38 \\
\hline & PSU 9 & 0.001 & 0.037 & 0.075 & 44.7 & 67.73 & 143.22 \\
\hline & PSU 10 & 0.002 & 0.059 & 0.103 & 26.2 & 45.96 & 65.88 \\
\hline & PSU 11 & 0.001 & 0.018 & 0.038 & 99.02 & 165.96 & 253.62 \\
\hline
\end{tabular}


vention models (mean reductions for 1 female every 10,20 and $50 \mathrm{yr}$ were $0.124 \pm 0.078,0.087 \pm 0.056$ and $0.049 \pm 0.034$, respectively), and all population simulations for translocations every 10 and $20 \mathrm{yr}$ produced inbreeding coefficients below 0.1. Inbreeding coefficients for donor populations were unaffected, and even in the 1 female every $10 \mathrm{yr}$ translocation model, the predicted increase after $250 \mathrm{yr}$ compared to the nointervention model was only $0.01 \pm 0.008$. Finally, all predicted values of $F_{\mathrm{ST}}$ remained significantly different from 0 , even in the every $10 \mathrm{yr}$ translocation model, ranging from 0.014 (PSU 5 versus PSU 11, 0.004 at $25 \mathrm{yr}^{2} 0.009$ at $100 \mathrm{yr} ; 0.014$ at $250 \mathrm{yr}$ ) to 0.028 (PSU 2 versus PSU 4, 0.038 at $25 \mathrm{yr}^{2} 0.033$ at $100 \mathrm{yr}, 0.028$ at

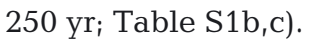

\section{Corridors}

For simplicity, corridor models simulated annual expansions in carrying capacity such that adjacent PSUs 1 and 3, 4 and 5 and 5 and 7 would reconnect after $100 \mathrm{yr}$, with the remainder reconnecting at the termination of the model, after $250 \mathrm{yr}$ (Appendix 1). Land purchase, forest matrix re-establishment rate, forest occupancy and usage by faunal populations are additional parameters not considered in the model and so conservative projections were used: reconnections and subsequent gene flow could potentially occur much more rapidly. Trends in gene diversity (Table S2) were very similar to those found for the 'no intervention' models, with the exception of PSUs 1, 3, 4, 5 and 7, where the demographic contact simulated at Year 100 concomitantly resulted in an increase in diversity. For example, in simulations of PSU 7, gene diversity declined to 0.947 of its original value by Year 25, to 0.939 by Year 100 and to 0.862 of its original value by
Year 250. While this decline is consistent, the final value is considerably higher than the 0.732 observed for the 'no intervention' model. Similar responses were observed for PSU 4 and PSU 3. In general, fewer simulations predicted extinction than in the no intervention model (Table S3 available at www.int-res.com/ articles/suppl/n012p249_supp.pdf), although PSUs 8 and 10 retained a $>5 \%$ probability of extinction at $250 \mathrm{yr}$ ( 0.061 and 0.118 , respectively). Table 3 shows the results for inbreeding coefficient and mean final population size. For the PSUs where reconnection was modelled to occur at $100 \mathrm{yr}$, large genetic gains (decreases in inbreeding coefficient) were evident. For example, for PSU 4, the simulated inbreeding coefficient after 250 yr was 0.042 as opposed to 0.133 predicted in the no intervention inbreeding model (trend 0.001 by Year $25 ; 0.033$ by Year $100 ; 0.042$ by Year 250 ) and similar gains were seen in PSU 7 (0.051 as opposed to 0.213 ). Fig. 2 shows the effect of corridor reconnection on the inbreeding coefficient in PSUs 4, 5 and 7. It is worth noting that the trend of increased inbreeding in PSU 7 is reversed at reconnection, in contrast to the more isolated PSUs 8 and 10, which were not modelled as reconnected until 250 yr. However, as Table 3 shows, a combination of relative isolation and small current carrying capacity means that PSUs 6, 8, 9 and 10 were still predicted to attain mean inbreeding coefficients $>0.1$ over the course of the $250 \mathrm{yr}$ simulation.

For PSUs simulated to reconnect after $100 \mathrm{yr}$, the increased carrying capacity led to a combined mean final population size for PSU 1 and PSU 3 of $382.02(K=$ 399 versus $K=338$ for PSU 1 and PSU 3 currently) and to a mean combined value of $380.26(K=461$ versus $K=$ 439) for PSUs 4,5 and 7. Other PSUs were predicted to respond positively to the increase in carrying capacity over $250 \mathrm{yr}$, with a mean final population size for all 10 PSUs of $1685.82(K=1913)$, an increase of $584(53 \%)$

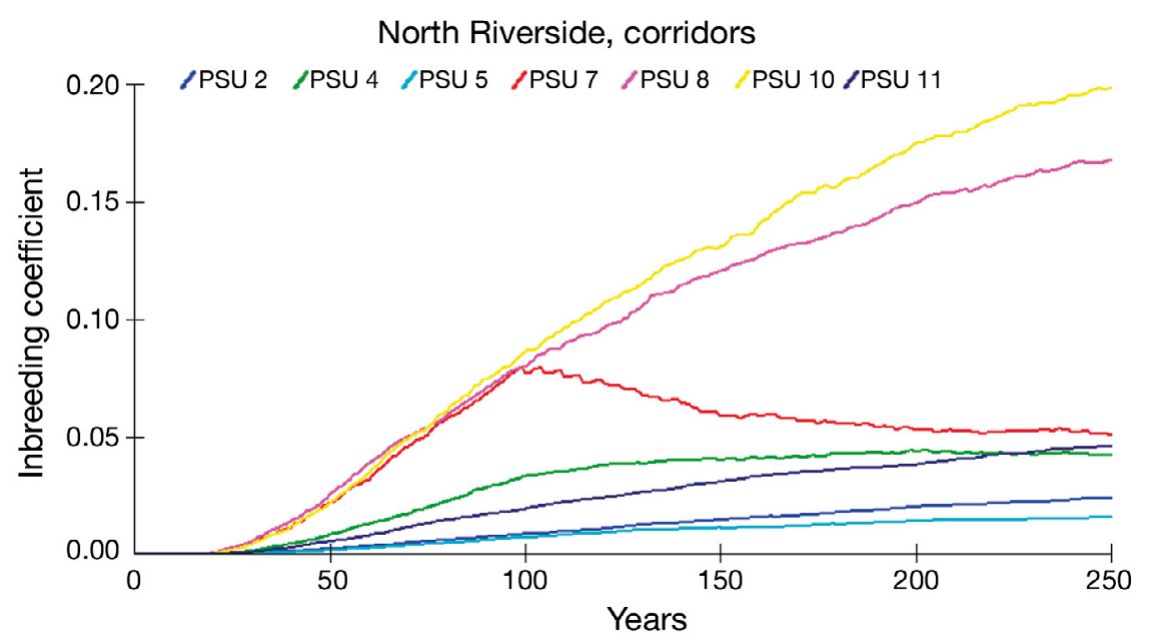

Fig. 2. Effect of corridor connection on within-primary sampling unit (PSU) inbreeding coefficients. PSUs 4,5 and 7 were modelled to reconnect after $100 \mathrm{yr}$ of the simulation; remaining PSUs were modelled to reconnect at $250 \mathrm{yr}$ 
over the current population size and $88 \%$ of predicted carrying capacity at the end of $250 \mathrm{yr}$. Trends in population size reflected changes in carrying capacity associated with corridor construction; for example, the most isolated PSU (9) showed an increase from 42.26 (Year 25) to 57.5 (Year 100) and 121.91 (Year 250). The latter value represents an approximately 4-fold increase over the result predicted for the no intervention, no inbreeding model. Although all unconnected PSUs remained significantly differentiated $(p<0.05)$ when $F_{\mathrm{ST}}$ values were estimated using Genepop v3.4, as expected, the reconnection events for PSUs 3, 4 and 7 produced lower pairwise $F_{\mathrm{ST}}$ values than the $100 \mathrm{yr}$ values in all 3 comparisons (Table S1c).

\section{Mixed approach}

Since translocation and corridors are not mutually exclusive options for the sanctuary, a combination of corridor establishment and a realistic translocation program was modelled of 1 female every 20 yr. Gene diversity trends at 25, 100 and 250 yr were equivalent to the ' 1 female every $20 \mathrm{yr}$ ' model (Table 3; Table S2), with mean final genetic diversity being higher in 9 of 11 PSUs. Mean final gene diversity was higher in all cases for the mixed model when compared with the corridor establishment model (Table 3; Table S2). In all cases, under the mixed model, the mean final probability of extinction was $<0.005$. Although the predicted mean inbreeding coefficients in this scenario were $<0.1$ after 250 yr for all PSUs (Table 3), the SD overlapped this value for PSUs 6, 8, 9 and 10. Table 3 shows the results of this approach. In comparison to the translocation-only and corridor-only simulations, the mixed simulation produced the highest mean final population size (1695), although this was not significantly higher than the corridors-only model. It also produced the lowest mean final inbreeding coefficient (0.06322), although 2 PSUs (7 and 10) exceeded a mean final inbreeding coefficient of 0.1 (0.104 and 0.103 , respectively), and this difference was not significantly lower than the translocation-only model. Furthermore, despite the combination of gene-flow and population size increase in the mixed model, all unconnected PSUs remained significantly differentiated at the $(\mathrm{p}<0.05)$ when $F_{\mathrm{ST}}$ was estimated from allele frequencies from 20 random simulations (Table S1d).

\section{DISCUSSION}

Despite the fact that several population viability models have previously been developed for orangutans (Ancrenaz 2003, Singleton et al. 2004), there are reasons for caution regarding the limitations of the model used here. Firstly, a single demographic model for orang-utans does not encapsulate the diversity of life history present within both currently recognised species, and it has been suggested that a cline in several key traits (anatomical, behavioural and ecological) exists from Sumatra to the northern tip of Borneo (Wich et al. 2009). While there is an unusually high degree of accuracy for much of the life history data in this model due to the extensive field studies carried out in the Kinabatangan, to date some elements of the model have been less easy to parameterise. Examples of these are the effects of density dependence, the additional mortality and reproductive cost associated with El Niño events and the assumption of equal mortality and reproductive rates in different PSUs, given that some populations may act as demographic sources and others as demographic sinks. These are long-term parameters which will take many years to accumulatelonger than many of the predicted extinction times for some PSUs in this model. However, since much of the sensitivity analysis for many orang-utan parameters has previously been carried out by Singleton et al. (2004), their relative importance for the outcomes of the model was already known.

The results of the no-intervention model suggest that the genetic and demographic viability of a number of subpopulations would be seriously compromised over the medium to long term, with up to 6 of 10 PSUs having a $>5 \%$ probability of extinction assuming inbreeding depression using pedigree-determined lethal equivalents after the 250 yr simulation. The interaction between small population size, demographic isolation and the subsequent effects of genetic drift and inbreeding have been demonstrated in numerous studies, although the empirical demonstration of these problems in wild great ape populations are absent from the literature to our knowledge. It is therefore a matter of debate as to whether inbreeding avoidance mechanisms (physiological, behavioural) may modify the predicted outcomes of these models, which do not include disassortative mating as a function of inbreeding or relatedness. However, in the case of the more isolated PSUs, if mating among relatives were to be avoided behaviourally, then such populations may become functionally extinct more rapidly than predicted here, as mate choice would become increasingly limited over time. Such populations are also expected to rapidly become genetically divergent (as seen in Table 2, where Wright's $F_{\mathrm{ST}}$ values were, in some cases, predicted to become very large over the time course of the simulation), and such a process may be expected to result in increasing incompatibility among gene pools. Currently there is no evidence that such genetic divergence impacts reproductive compat- 
ibility in this population, but natural demographic isolation between the populations north and south of the river has already been demonstrated (Goossens et al. 2005, Jalil et al. 2008), and conservative conservation management would normally seek to maintain this demographic feature (indeed we recommend that these riverside populations be maintained as separate Management Units as long as this is practically feasible) given the significantly greater $F_{\mathrm{ST}}$ values among riversides than within (Moritz 1994, Goossens et al. 2005, Jalil et al. 2008).

If the very high extinction probabilities for some PSUs (especially 6 to 10; Table 1) would seem to suggest that non-interventive approaches are not a viable conservation option, especially given the assumption of no carrying capacity degradation over time for these small forest patches, then the question arises as to what is the most efficient management approach from a biological, ecological, financial and logistical standpoint. For financial and logistical (and possibly biological) efficiency, translocation of individuals among PSUs or from other sources is an attractive option. Because of the long generation time in this species, relatively modest translocation frequencies could potentially provide significant demographic and genetic augmentation to recipient populations and may ameliorate the effects of inbreeding and genetic drift within populations and genetic differentiation among them.

However, translocation as a management tool is not without risk. Evidence from other sites where rehabilitants have been reintroduced into wild populations suggests that it is a potentially successful approach (e.g. Utami et al. 2002), although data remain largely uncollated. The impact on both source and sink populations from the point of view of social structure, animal health and accidents during translocation must be considered, and any reintroductions must comply with IUCN guidelines. The sex we chose to model for translocated individuals was female, largely because the genetic benefits of translocating females is predicted to be the highest on average since females have a lower reproductive skew (Utami et al. 2002, Goossens et al. 2006b).

A key element of such a management strategy is that there are viable demographic sources of potential immigrants for translocation. Here, we simulated the use of the 3 largest PSUs as the source. The rationale for this choice was based on practicalities of the situation in the Kinabatangan area. We chose the largest populations, as they seemed the most obvious choice but also because of their geographic location, which made them a natural choice to serve as sources to neighbouring populations. However, our simulations showed that 2 of these are expected to decrease demographically even in the no intervention scenario (PSU 1 and PSU 5, Table 1). This result may have significant practical implications, since it shows that large populations may not always be the most likely to be demographically stable. However, even if a population is not expected to grow based on PHVA simulations, the reality of the local situation may still require this population to serve as a source, either because of its geographic location or because it is large enough to suffer less from a decrease than smaller candidate source populations. However, in the Kinabatangan, other solutions may also be feasible. For instance, individuals rescued from plantations and human settlements in the surrounding area over the coming years (a regular occurrence) and from the rehabilitation centre at Sepilok (Sandakan, Sabah) may provide an additional source of genetic and demographic stability.

Movement of individuals every $50 \mathrm{yr}$ did not prevent the accumulation of significant amounts of inbreeding within the PSUs having the smallest carrying capacity but the more frequent scenarios were more successful at controlling inbreeding coefficients in these populations. Interestingly, in no case did the model predict a subsequent lowering of $F_{\mathrm{ST}}$ values to that below the threshold of significance, possibly due to the slow reproductive rate of females and the stronger sampling effects in small populations. However, it is important to stress that the model used does not account for possible genetic gains that would be obtained through the arrival of new immigrants. It has been demonstrated that a few immigrant genomes can potentially have a profound effect on the genetic diversity and fitness of a subpopulation (e.g. Keller et al. 1994, 2001, Saccheri \& Brakefield 2002), either through disproportionately high reproductive success due to mate choice in favour of genome dissimilarity (which could not be modelled here) or because the life history strategy of immigrant individuals increases their lifetime reproductive success. Therefore, the models used here are likely to underestimate the genetic gains of immigration both at the level of within-population inbreeding and amongpopulation genetic differentiation. However, a number of problems exist with the utilisation of this management approach alone. First, it poses a substantial demographic challenge to the donor populations, especially if, as here, there are 1 or 2 large populations donating to several or many small sink populations (e.g. PSU 1 in the 1 translocation every 10 yr scenario modelled here). Second, it does not address the ongoing ecological issues associated with maintaining viable habitat over the period of the management plan.

Creation of habitat corridors and ecological restoration are regarded as 2 very important components of long-term sustainable conservation management. However, they are sometimes extremely difficult to carry out for logistical and financial reasons. Habitat 
corridors among PSUs of the LKWS are currently under construction at small scales and so we considered their potential impact on population demographic and genetic viability here. Many factors can influence the timescale and demographic gains associated with forest corridor establishment, such as financial constraints (land purchase), forest re-establishment rates (which are slow for high canopy dipterocarp forest but which can be much quicker for riparian and seasonally inundated forest) and habitat occupancy and corridor usage dynamics by the faunal community, which could in principle be almost instantaneous or could be a protracted process. Since these factors are currently unknown, we took an extremely conservative approach in terms of the timescales involved, and our results therefore need to be treated with caution, especially considering the not inconsiderable difficulties involved with creating connections across human habitation and roads (although artificial rope bridges across tributaries offer a potential solution at smaller scales). However, creating habitat corridors linking the smaller PSUs upstream in the Kinabatangan will be extremely challenging and is unlikely to occur over short timescales.

The demographic and genetic benefits of incremental carrying capacity increases and genetic reconnection are clear in those populations where population sizes and carrying capacities are currently small and where the gains are proportionately highest (e.g. PSU 7, Fig. 2). The eventual reconnection of all PSUs to create a contiguous habitat corridor within the LKWS is a stated aim of the developing management plan for the sanctuary. However, the final outcome will crucially depend on the speed at which reconnection can take place. In our models we tried to use realistic scenarios, which show clearly that for the most isolated PSUs, corridor reconnection is unlikely to occur rapidly enough to prevent large-scale genetic and demographic loss and alone may not be able to prevent extinction in these important populations. If, as genetic evidence currently suggests (Jalil et al. 2008), the Kinabatangan floodplain was originally colonised from the Crocker Range/Mt. Kinabalu massif when the lowland forest re-established following the Pleistocene, then the upstream orang-utan populations, including those inside the sanctuary, are likely to possess the highest levels of genetic diversity, and indeed Jalil et al. (2008) found some evidence for this with mitochondrial DNA. Thus, the upstream LKWS populations, although demographically more limited, possess substantial unique genetic diversity which would be lost if they went extinct.

Therefore, if neither translocation nor corridor establishment alone are likely to solve all of the problems for the populations within the sanctuary, a mixed ap- proach may fulfil this role. Our mixed model identified the most logistically feasible translocation program, which produced the biggest proportional change in results as the 1 female every 20 yr scenario. In combination with the corridor re-establishment scenario, this approach produced the largest mean final population size and the lowest mean inbreeding coefficient across the LKWS and potentially provides both a realistic and effective solution to the current orang-utan demographic problem.

Much debate has occurred in the literature about the demographic and genetic thresholds needed to create long-term viable populations for conservation; however, there is significant support for the so-called 50:500 (effective population size:census size) rule as a pragmatic approach to minimum viable population (MVP) conservation targeting. The current estimated Ne for PSUs 1 and 2 north and south of the river is 30 (Goossens et al. 2006), producing an Ne:N ratio of approximately 1:7. Since the census size is estimated at 728 on the north riverbank and 377 on the south riverbank, this could equate to an effective population size of approximately 100 north of the river and 50 south of the river, assuming an additive increase in Ne on each riverside when all individuals are taken into consideration. Assuming that demographic gains from carrying capacity augmentation and translocation brings a proportionate gain at the genetic level (and this could be even more efficient if appropriately selected individuals from outside the LKWS are used for demographic augmentation), corridor reestablishment under the conditions modelled here is predicted to yield a carrying capacity of 1915, equivalent to an $\mathrm{Ne}$ of 273 (approximately 180 north of the river and 90 south of the river). Although no simulations achieved 1915 over the $250 \mathrm{yr}$ term of the model, this may be due to the incremental dynamics of the corridor reestablishment and could also reflect the slow demographic dynamics of this species. Nonetheless, additional augmentation could be used from outside the LKWS at strategic points to enable a population size of approximately 2000 individuals to be attained and long-term demographic viability to be secured.

Acknowledgements. We thank the Economic Planning Unit and especially P. Munirah for granting permission to carry out research in Sabah. This work was funded by the Darwin Initiative for the Survival of Species (grant no. 09/016, DEFRA, UK), Cardiff University, Universiti Malaysia Sabah and HUTAN. Orang-utan hair and faeces were exported to the UK under export permit from the Federation of Malaysia (CITES Certificate no. 0467, security stamp No. MY 9123707) and under import permit from the UK (CITES Certificate nos. 236719/01 for shed hair samples and 236719/02 for faecal samples). This paper is dedicated to the memory of Mohedi Andau, whose inspiration and collaboration was key to the inception and execution of this project. 


\section{LITERATURE CITED}

Ancrenaz M (ed) (2003) Proceedings of the International Workshop on orang-utan in Sabah, 25-27 August 2003, Kota Kinabalu, Sabah

Ancrenaz M, Goossens B, Gimenez O, Sawang A, LackmanAncrenaz I (2004) Determination of ape distribution and population size using ground and aerial surveys: a case study with orang-utans in lower Kinabatangan, Sabah, Malaysia. Anim Conserv 7:375-385

Asquith NM (2001) Misdirections in conservation biology. Conserv Biol 15:345-352

Bouchy P, Theodorou K, Couvet D (2005) Metapopulation viability: influence of migration. Conserv Genet 6:75-85

Bowen BW, Roman J (2005) Gaia's handmaidens: the Orlog model for conservation biology. Conserv Biol 19: 1037-1043

Frankham R (2003) Genetics and conservation biology. C R Biol 326 (Suppl 1):S22-S29

- Goossens BG, Chikhi L, Utami SS, de Ruiter JR, Bruford MW (2000) Multiple-samples and multiple-extracts approach for microsatellite analysis of faecal samples. Conserv Genet 1:157-162

Goossens B, Chikhi L, Jalil MF, Ancrenaz M and others (2005) Patterns of genetic diversity and migration in increasingly fragmented and declining orang-utan (Pongo pygmaeus) populations from Sabah, Malaysia. Mol Ecol 14:441-456

> Goossens B, Chikhi L, Ancrenaz M, Lackman-Ancrenaz I, Andau P, Bruford MW (2006a) Genetic signature of anthropogenic population collapse in orang-utans. PLoS Biol 4:e25

Goossens B, Setchell JM, James SS, Funk SM and others (2006b) Philopatry and reproductive success in Bornean orang-utans (Pongo pygmaeus). Mol Ecol 15:2577-2588

Jalil MF, Cable J, Sinyor J, Lackman-Ancrenaz I, Ancrenaz M, Bruford MW, Goossens B (2008) Riverine effects on mitochondrial structure of Bornean orang-utans (Pongo pygmaeus) at two spatial scales. Mol Ecol 17:2898-2909

Keller LF, Waller DM (2002) Inbreeding effects in wild populations. Trends Ecol Evol 17:230-241

Keller LF, Arcese P, Smith JNM, Hochachka WM, Stearns SC (1994) Selection against inbred song sparrows during a natural population bottleneck. Nature 372:356-357

Keller LF, Jeffery KJ, Arcese P, Beaumont MA, Hochachka WM, Smith JNM, Bruford MW (2001) Immigration and the ephemerality of a natural population bottleneck: evidence from molecular markers. Proc Biol Sci 268:1387-1394

Keyghobadi N, Roland J, Matter SF, Strobeck C (2005) Among- and within-patch components of genetic diversity respond at different rates to habitat fragmentation: an empirical demonstration. Proc Biol Sci 272:553-560

Lackman-Ancrenaz I, Ancrenaz M, Saburi R (2001). The Kinabatangan orang-utan conservation project. The apes: challenge for the 21st century. Conf Proc, 10-13 May 2000, Brookfield, IL, p 262-265

Lacy RC, Borbat M, Pollak JP (2005) VORTEX: a stochastic simulation of the extinction process. Version 9.50. Chicago Zoological Society, Brookfield, IL

Madsen T, Shine R, Olsson M, Wittzell H (1999) Restoration of an inbred adder population. Nature 402:34-35

Madsen T, Ujvari B, Olsson M (2004) Novel genes continue to enhance population growth in adders (Vipera berus). Biol Conserv 120:145-147

Miller PS, Lacy RC (2005) VORTEX: a stochastic simulation of the extinction process. Version 9.50 user's manual. Conservation Breeding Specialist Group (SSC/IUCN), Apple Valley, MN

Moritz C (1994) Defining Evolutionary Significant Units for conservation. Trends Ecol Evol 9:373-375

Raymond M, Rousset F (1995) Genepop (version 1.2) - population genetics software for exact tests and ecumenicism. J Hered 86(3):248-249

Reed JM, Mills LS, Dunning JB, Menges ES and others (2002) Emerging issues in population viability analysis. Conserv Biol 16:7-19

> Robert A, Couvet D, Sarrazin F (2003) Bottlenecks in large populations: the effect of immigration on population viability. Evol Ecol 17:213-231

Saccheri IJ, Brakefield PM (2002) Rapid spread of immigrant genomes into inbred populations. Proc R Soc Lond Ser B Biol Sci 269: 1073-1078

Saccheri I, Kuusaari M, Kankare M, Vikman P, Fortelius W, Hanski I (1998) Inbreeding and extinction in a butterfly metapopulation. Nature 392:491-494

> Schwartz MK, Mills LS (2005) Gene flow after inbreeding leads to higher survival in deer mice. Biol Conserv 123: 413-420

Singleton I, Wich S, Husson S, Stephens S and others (eds) (2004) Orangutan population and habitat viability assessment: final report. IUCN/SSC Conservation Breeding Specialist Group, Apple Valley, MN

> Stockwell CA, Hendry AP, Kinnison MT (2003) Contemporary evolution meets conservation biology. Trends Ecol Evol 18:94-101

Talbot SL, Pearce JM, Pierson BJ, Derksen DV, Scribner KT (2003) Molecular status of the dusky Canada goose (Branta canadensis occidentalis): a genetic assessment of a translocation effort. Conserv Genet 4:367-381

Tallmon DA, Luikart G, Waples RS (2004) The alluring simplicity and complex reality of genetic rescue. Trends Ecol Evol 19:489-496

Utami SS, Goossens B, Bruford MW, de Ruiter JR, van Hooff JARAM (2002) Male bimaturism and reproductive success in Sumatran orang-utans. Behav Ecol 13(5):643-652

Wich SA, Utami Atmoko SS, Setia TM, van Schaik CP (2008) Orangutans: geographic variation in behavioural ecology and conservation. Oxford University Press, Oxford 
Appendix 1. General parameter values for Lower Kinabatangan Wildlife Sanctuary orang-utans (based on Ancrenaz 2003, Singleton et al. 2004). EV: environmental variance; N: population size; K: carrying capacity

- Extinction defined as no animals of 1 or both sexes

- Inbreeding depression (where used): 4.06 lethal equivalents per genome (Singleton et al. 2004), 50\% of genetic load contributed by lethals

- Natural dispersal not modelled

- EV in mortality is concordant among age-sex classes, but independent from $\mathrm{EV}$ in reproduction

- Correlation of EV among populations = 1

- First age of reproduction for females: $15 \mathrm{yr}$; for males: $18 \mathrm{yr}$

- Maximum breeding age (senescence): 45 yr

- Sex ratio at birth (percent males): 55

- Polygynous mating

- Percentage of adult males in the breeding pool $=100$

- Percentage of adult females breeding $=\{20.5-[(20.5-10)$ $\left.\left.\times(\mathrm{N} / \mathrm{K})^{2}\right]\right\} \times[\mathrm{N} /(1+\mathrm{N})]=$ quadratic density dependence with no Allee parameter (Singleton et al. 2004)

- Variance in percentage of adult females breeding: $\mathrm{SD}=10$

- $100 \%$ of females produce 1 progeny

- Percentage mortality of females between ages 0 and $1=5$ $(\mathrm{SD}=2.5)$; between ages 1 and $2=6(3)$; between ages 3 and $7=0.5(0.25)$; between ages 8 and $10=6(3)$; between ages 11 and $15=0.5(0.25)$; between ages 16 and $45=1.75(0.88)$

- Percentage mortality of males between ages 0 and $1=5$ $(\mathrm{SD}=2.5)$; between ages 1 and $2=5(2.5)$; between ages 2 and $7=3(1.5)$; between ages 8 and $10=6(3)$; between ages 11 and $14=0.5(0.25)$; between ages 15 and $45=1.25(0.63)$
- Two catastrophes: adult mortality as a result of crop raiding (frequency: $17 \%$; multiplicative effect on reproduction $=1$; multiplicative effect on survival $=0.98$ ) and severe drought (frequency: $8 \%$; multiplicative effect on reproduction $=1$; multiplicative effect on survival $=$ 0.965)

- Initial population size - see Ancrenaz et al. (2004), their Table 2: primary sampling unit $($ PSU 1$)=230$; PSU $2=$ 209; PSU $3=63$; PSU $4=61 ;$ PSU $5=293$; PSU $6=55$; PSU $7=23 ;$ PSU $8=22 ;$ PSU $9=49 ;$ PSU $10=23 ;$ PSU $11=97$

- Age distribution from Ancrenaz (2003) on the basis of 71 observations made by boat. Juveniles $=10 \%(50: 50$ ratio); adult females $=26 \%$; adult males lacking secondary sexual characteristics $=27 \% ; 37 \%$ 'flanged' adult males. Individuals allocated evenly to different ages across all age classes and we assumed that 'unflanged' males had the same age distribution as 'flanged' males

- Carrying capacity (modified from Ancrenaz 2003 to reflect present field conditions): PSU $1=230$; PSU $2=$ 351; PSU 3 = 108; PSU 4 = 72; PSU $5=313$; PSU $6=62$; PSU $7=54 ;$ PSU $8=97 ;$ PSU $9=49 ;$ PSU $10=31 ;$ PSU $11=120$

- Trend in carrying capacity for corridor models: PSU $1=$ $+0.1 \%$; PSU $2=+0.2 \%$; PSU $3=+0.8 \%$; PSU $4=$ $+0.5 \%$; PSU $5=+0.1 \%$; PSU $6=+0.8 \%$; PSU $7=$ $+0.1 \% ;$ PSU $8=+1 \% ;$ PSU $9=+1.6 \%$; PSU $10=+2 \%$; PSU $11=+2 \%$ 